\title{
Les marges de l'analyse du discours philosophique en question : le cas Derrida
}

Questioning the Margins of Philosophical Discourse Analysis: The Case of Jacques Derrida

\section{Charlotte Thevenet}

\section{(2) OpenEdition}

\section{Journals}

Édition électronique

URL : http://journals.openedition.org/aad/3138

DOI : 10.4000/aad.3138

ISSN : $1565-8961$

Éditeur

Université de Tel-Aviv

Référence électronique

Charlotte Thevenet, «Les marges de l'analyse du discours philosophique en question : le cas Derrida », Argumentation et Analyse du Discours [En ligne], 22 | 2019, mis en ligne le 15 avril 2019, consulté le 23 septembre 2019. URL : http://journals.openedition.org/aad/3138 ; DOI : 10.4000/aad.3138

Ce document a été généré automatiquement le 23 septembre 2019.

Argumentation \& analyse du discours est mis à disposition selon les termes de la licence Creative Commons Attribution - Pas d'Utilisation Commerciale - Pas de Modification 4.0 International. 


\section{Les marges de l'analyse du discours philosophique en question : le cas Derrida}

Questioning the Margins of Philosophical Discourse Analysis: The Case of Jacques Derrida

Charlotte Thevenet

1 L'œuvre de Derrida semble au premier abord mettre l'analyse du discours philosophique face à ses limites ; des textes stéréographiques ${ }^{1}$ comme Glas, « Survivre ", "Tympan », Feu la cendre, ou encore "Circonfession ", qui exposent un dédoublement de l'énonciation, défient l'analyste d'y trouver la voix du philosophe, l'énoncé d'une doctrine, l'exposition d'une méthode. Ces textes doubles, à première vue atypiques par

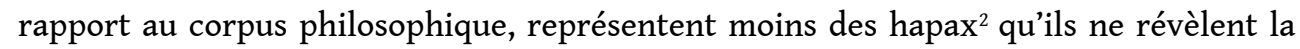
volonté constante et traçable dans toute l'œuvre derridienne d'exposer les textes dans leur illisibilité, leur « imprenab[ilité] »; " (Ah) tu es imprenable (eh bien) reste » lit-on dans Glas (Derrida 1974: 77b), prolepse qui anticipe la réaction du lecteur lui-même, découragé de ne pouvoir assimiler les deux colonnes en même temps. Mais des textes monographiques et linéaires posent au moins autant de difficultés de lecture : ainsi l'ouvrage consacré à Éperons par le Groupe de Recherche sur l'analyse du discours Philosophique (GradPhi) est-il justement intitulé «Lire Derrida?», montrant bien la gageure que peut représenter la lecture analytique d'un tel texte (Maingueneau et Vallespir 2015). L'œuvre derridienne ne serait-elle alors analysable que selon ses propres termes, au risque de lectures infiniment mimétiques ${ }^{3}$ et volontiers circulaires ? Entrer dans le texte derridien semble exiger du critique qu'il abandonne sur le seuil ses outils d'analyse, ses grilles de lecture et ses présupposés méthodologiques, pour ne pas défigurer la philosophie qui s'écrit là et qui consiste en une infinie déconstruction de son champ même (la métaphysique). Dans cette perspective, l'analyse du discours philosophique constituerait un arsenal théorique et méthodologique non seulement hétérogène à la philosophie de Derrida, mais encore, et bien qu'elle ne les reconduise pas de manière classique, réutilisant des catégories que l'œuvre derridienne se serait 
attachée à déconstruire (le livre, le sujet...) $)^{4}$. L'analyse du discours philosophique serait donc doublement caduque pour traiter du corpus derridien : ses catégories seraient inadéquates pour saisir les opérations discursives, et de surcroît, tenter seulement de saisir le discours philosophique derridien à travers ces catégories relèverait d'une erreur de lecture initiale. Le corpus derridien apparaît donc marqué d'un «Défense d'entrer » à l'égard de l'analyse du discours philosophique.

Certains éléments invitent pourtant à nuancer ce premier constat: loin d'être incompatibles, la philosophie de Derrida et l'analyse du discours philosophique entretiendraient un rapport d'affinité. Outre leur contemporanéité, elles partagent un intérêt pour la façon dont la philosophie s'écrit, et non simplement pour ce qu'elle écrit, distinction artificielle entre forme et contenu, style et doctrine qu'elles s'attachent toutes deux à ne pas reconduire. Derrida, qui s'inscrit là dans le sillage de Husserl et de Heidegger, s'intéresse au "discours philosophique » à la fois comme champ d'investigation et comme pratique. Ainsi les cours donnés à l'ENS de 1969 à 1971 témoignent-ils de cette préoccupation : l'année 1969-1970 est consacrée à « Théorie du discours philosophique 1 : la métaphore dans le discours philosophique ", travail publié en 1972 dans Marges de la philosophie sous le titre « La mythologie blanche", tandis que l'année 1970-1971, on le sait moins, s'intitulait «Théorie du discours philosophique 2. La forme du texte philosophique: les conditions d'inscription du texte de philosophie (l'exemple du matérialisme)» (Mercier 2018). On peut également citer le cours de l'année 1972, "Philosophie et rhétorique au XVIIIe siècle : Condillac et Rousseau » qui donnera lieu à deux manuscrits : Archéologie du frivole, publié en 1973, et « Le calcul des langues ", texte inachevé en deux colonnes à ce jour inédit. Citons enfin le sous-titre d' Éperons : «les styles de Nietzsche » $(1976,1978)$, et son premier titre : «La question du style» (1973)5, qui trahissent la préoccupation du philosophe pour le discours philosophique, particulièrement vive entre 1969 et 1978. La philosophie de Derrida et l'analyse du discours philosophique s'intéressent donc à la même chose : la discursivité de la philosophie, les conditions de possibilité de son énonciation et de sa réception.

3 C'est le paradoxe d'une hétérogénéité apparente et d'affinités réelles entre la philosophie de Derrida et l'analyse du discours philosophique que je souhaite travailler ici, en confrontant les textes stéréographiques de Derrida aux catégories de l'analyse du discours philosophique. Un tel corpus permet en effet d'aborder les textes les plus évidemment préoccupés par la question du discours de la philosophie - étant entendu que la question traverse toute l'œuvre de Derrida. J'interrogerai d'abord les supposées marges du discours philosophique : celles dans lesquelles celui de Derrida s'expose, et celles dans lesquelles l'analyse du discours philosophique semble paradoxalement l'appréhender. J'esquisserai ensuite la forme des affinités qui relient la philosophie derridienne et l'analyse du discours philosophique à travers l'examen de leur commune inscription du biographique dans le discours philosophique. Enfin, un bref examen de la façon dont circulent voix et écriture dans certains textes stéréographiques de Derrida me permettra de proposer une redéfinition de la notion de ton, qui, ainsi informée par le travail de la philosophie derridienne, pourrait permettre d'enrichir l'arsenal de l'analyse du discours philosophique. 


\section{Interroger les marges du discours philosophique : la tradition de la déconstituance}

\subsection{Une œuvre aux « marges de la philosophie »}

$4 \quad$ La difficulté qui se présente immédiatement quand on aborde ensemble la philosophie de Derrida et l'analyse du discours philosophique ${ }^{6}$, c'est que la première semble d'abord subvertir la seconde. Or, il s'agit davantage d'un rapport complexe que d'un renversement ou d'une mise en échec pure et simple. La tentation de voir dans l'œuvre de Derrida une entreprise de subversion de toute tentative analytique qui lui serait hétérogène vient d'abord du statut liminaire que se donne l'œuvre derridienne ellemême : c'est en effet son appartenance à «la philosophie » qui semble sans cesse mise en question.

5 Outre le titre, explicite, de Marges de la philosophie, on peut noter combien la confrontation avec la littérature exhibe une apparente (et à mon sens trompeuse) subversion des disciplines et des catégories génériques. La littérature intervient à deux titres au moins dans le corpus derridien; d'une part, elle produit des objets dignes de commentaire, comme l'œuvre de Ponge dans Signéponge (1984), ou celle de Genet dans Glas $^{7}$ (1974); d'autre part, elle sert de contre-champ au texte philosophique qu'il s'agisse du texte de Derrida lui-même comme dans « Tympan » (1972b), ou de celui d'un autre comme dans «La double séance» (1972a). Dans les deux cas, la littérature fonctionne comme un facteur de marginalisation de la philosophie : le texte littéraire marque la philosophie au sceau de son hétérogénéité et peut sembler en brouiller les limites plutôt que les assurer. Encore une fois : c'est bien la question de l'appartenance de l'œuvre derridienne à la philosophie qui est en jeu, autant dans son écriture ellemême que dans sa réception ${ }^{8}$. Si l'œuvre de Derrida parait d'abord inanalysable du point de vue de l'ADPhi, c'est tout simplement qu'elle paraît ne pas appartenir en propre à la philosophie ${ }^{9}$. Or, on ne saurait sérieusement considérer les disciplines et champs du savoir comme des cuves dont l'étanchéité préviendrait toute communication entre leurs contenus ; parmi les contemporains de Derrida, qu'on pense à Deleuze et à ses nombreux commentaires d'œuvres littéraires (par exemple, La Vénus à la fourrure, 2007), à Foucault analysant les Ménines de Vélasquez (2005), à Lacan, enfin, qui semble avoir couvert toute la littérature depuis la tradition courtoise jusqu'à Poe, nombreux sont ceux qui ne se sont pas cantonnés aux objets appartenant strictement à leur discipline. Pourtant, personne, à ma connaissance, ne songe à faire de Foucault un historien de l'art, ou de Lacan un critique littéraire. Pourquoi l'infusion d'une dimension littéraire dans l'écriture et la réflexion philosophique par Derrida estelle si discriminante dans la définition générale de son œuvre, à l'inverse d'autres exemples? Mon hypothèse est la suivante: c'est l'association d'un questionnement permanent sur ce qui fait la philosophie et d'une lecture de textes littéraires qui produit l'effet d'une subversion des normes disciplinaires.

6 S'intéressant à ce questionnement lui-même (qu'est-ce qui fait qu'un texte est de la philosophie?), on voit qu'il est particulièrement mis en valeur dans les textes atypiques. "Tympan ", qui doit servir de préface à Marges de la philosophie (1972), est paradigmatique de la rhétorique derridienne; j'aimerais en souligner deux traits : sa dimension anti-systématique, et son positionnement paradoxal par rapport à «la philosophie». "Tympan» commence par un exergue en forme de «tympan» 
architectural : trois citations de Hegel sont superposées les unes aux autres, trois blocs de texte disposés de manière pyramidale, qui parlent justement de la philosophie et de sa limite.

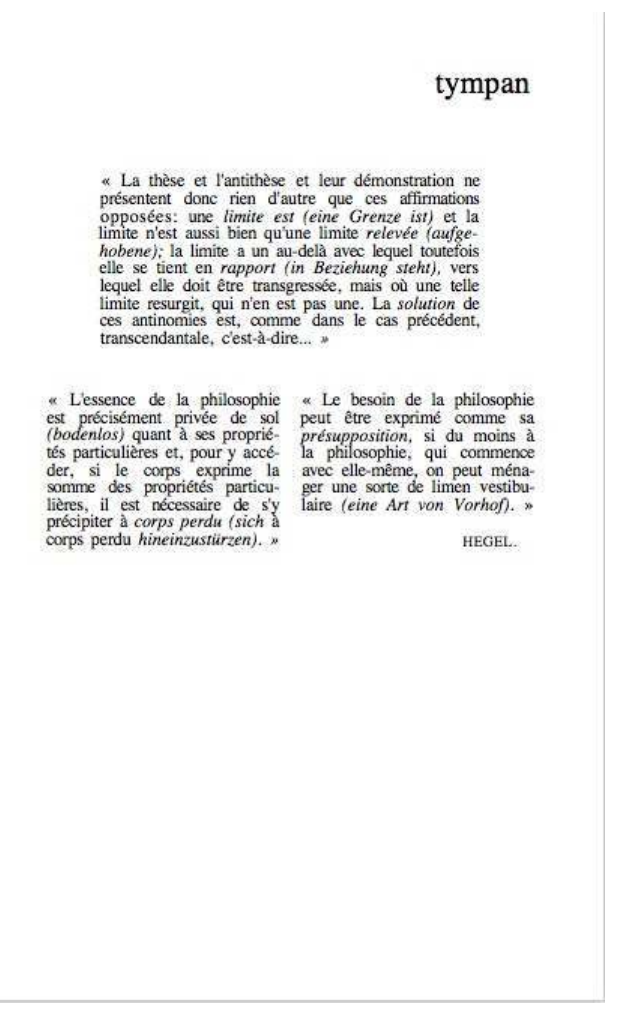

Page de titre de «Tympan » dans Marges de la philosophie. Crédits : Charlotte Thevenet

7 La disposition des blocs de texte semble mimer de manière parodique le titre de l'essai consacré à Hegel publié dans le même ouvrage, "Le puits et la pyramide", mais également le rythme tripartite de la philosophie hegelienne et sa fameuse "relève » (Aufhebung), présente dans le rectangle supérieur. Ce que semble annoncer cette page liminale, c'est bien la possibilité d'écrire aux marges de la philosophie, ni dedans ni dehors, de produire une « limite » qui ne serait pas « relevée », et ainsi de multiplier les seuils: l'introduction se double d'un exergue, qui traite lui-même de la limite, du commencement et du «limen vestibulaire", comme si tous ces frontispices étaient voués à s'engendrer et à se réfléchir les uns les autres ad libitum. L'effet produit est net : Marges de la philosophie est un ouvrage de philosophie non-systématique, plutôt la déconstruction des rouages de la métaphysique occidentale que sa continuation bien huilée. Corollaire de ce positionnement anti-systématique, la philosophie de Derrida exhibe une relation paradoxale, problématique même à la philosophie. Ainsi de « Tympan », encore, qui s'ouvre sur ces mots : « Tympaniser - la philosophie » (1972b : I) ; non seulement l'ouvrage adopte-t-il un positionnement anti-systématique, mais il fait montre, dès le texte d'introduction, d'une irrévérence rare par rapport à la discipline même dans laquelle il s'inscrit. Si «tympaniser " signifie " faire connaître à grand bruit", son deuxième sens, "décrier hautement quelqu'un, déclamer contre lui », est porteur d'une dimension polémique, tandis que la forme réfléchie du verbe, "se tympaniser", signifiant "être ridicule", introduit satire et parodie dans la démarche philosophique ${ }^{10}$. Cette désinvolture est doublement étonnante: d'abord, parce qu'il s'agit d'une préface, lieu traditionnel d'une captatio benevolentiae qui se fait 
ici paradoxale ; ensuite, parce qu'on attendrait d'un texte de philosophie qu'il exhibe sa légitimité à s'inscrire dans ce champ plutôt qu'il ne mette en scène ses velléités à le tourner en ridicule. L'œuvre de Jacques Derrida, et en particulier ses textes stéréographiques, semblent donc mal se prêter à l'ADPhi tant leur appartenance à la philosophie semble incertaine; ces textes sont construits pour produire l'effet d'une perturbation des normes disciplinaires et par conséquence analytiques, invitant à une lecture en termes de subversion.

\subsection{La déconstituance aux marges de l'ADPhi}

8 Le paradoxe posé par une philosophie difficilement identifiable qui questionne sa propre discipline et par là sa propre appartenance disciplinaire n'a pas échappé à Cossutta et Maingueneau. S'ils ont montré, dans leur article fondateur, que le discours philosophique appartenait à la catégorie des discours constituants, dont la spécificité est de «fonder et de n'être pas fondé » (Maingueneau et Cossutta 1995 : 112), ils attirent presque immédiatement l'attention sur le fait que certaines philosophies entretiennent un rapport plus problématique à leur propre légitimité. L'ADPhi s'inscrit en effet dans une "analyse des discours constituants » (idem), c'est-à-dire l'analyse de «types de paroles qui font autorité, qui sont reconnues comme pouvant donner sens aux actes de l'ensemble de la collectivité " (Maingueneau 2015 : 25). Ces discours ne tiennent leur autorité que d'eux-mêmes: dans un discours constituant, "l'énonciation se déploie comme dispositif de légitimation de l'espace de sa propre énonciation, à l'articulation d'un texte et d'une manière de s'inscrire dans un univers social» (Maingueneau et Cossutta 1995: 115) ${ }^{11}$. La spécificité du discours philosophique à l'égard d'autres discours constituants réside dans

la prétention [à] s'auto-constituer et [à] jouer un rôle constituant à l'égard d'autres régimes discursifs [...]. La spécificité du discours philosophique parmi les discours constituants est d'être le discours qui veut expliciter les conditions de possibilité de toute constitution discursive (Cossutta $1995: 16$ ).

Or, tout un corpus de discours philosophiques se distingue radicalement de cette définition, et l'ADPhi comme discours constituant a eu à réfléchir, dès son élaboration, à ces discours qui, loin de " ne s'autoriser que d'eux-mêmes " (Maingueneau $2015: 26$ ), de prétendre à une position de surplomb par rapport à d'autres et de se mettre en scène comme "Source légitimante" (idem) d'eux-mêmes, se montraient comme constitués plutôt que constituants, parole subversive plutôt qu'autoritaire.

Chacun des deux auteurs accorde une place différente à ces discours au sein de l'ADPhi : Cossutta parle de discours «destituants» tandis que Maingueneau subsume une distinction entre régime non marqué et régime marqué de discours sous la catégorie de " constituance ». Malgré l'attention portée à l'hétérogénéité du discours philosophique, il me semble que, dans les deux cas, les auteurs reconduisent la marginalité paradoxale montrée du discours philosophique derridien au sein des catégories qu'ils forgent pour le comprendre. De la même façon que Derrida produit un discours philosophique qui se montre comme étant aux "marges » supposées de la philosophie tout en ne quittant jamais une critique de la métaphysique toute philosophique, Cossutta et Maingueneau font droit à des discours paradoxaux au sein du discours philosophique tout en les reléguant en permanence à la marge d'un régime dominant (discours auto-constituant pour Cossutta, régime non marqué pour Maingueneau). 
11 Pour Cossutta, alors que le discours philosophique dans son acception générale est conçu comme un discours qui trouve en lui-même sa propre légitimité à accéder à la parole philosophique (auto-constituant), certaines philosophies fonctionnent paradoxalement sur une logique « destituante », selon laquelle la parole philosophique est mise au service d'une délégitimation de la parole philosophique même. Cossutta identifie, à côté du discours philosophique auto-constituant, une lignée destituante, qui va des sceptiques à Derrida, et qui oblige le lecteur à « devoir résoudre la difficulté d'une discursivité auto-contradictoire" (Cossutta 2015b). Or, il me semble que cette tradition occupe une place ambivalente dans l'ADPhi. D'une part, elle est marginale, ce qui reproduit la place que ces philosophies se donnent à elles-mêmes par rapport à la philosophie; en effet, la question du scepticisme ou plus largement des philosophies déconstituantes intervient toujours en regard, mais non sur le même plan, que celle de la constituance dont elle me semble pourtant solidaire. Ainsi l'article "Les discours constituants vingt ans après " consacre-t-il de longs passages au problème posé à la catégorie de discours constituant par "l'hypothèse sceptique " (Cossutta 2015b); et si l'auteur reconnaît que ces philosophies «ne sont pas pour autant extérieures à la philosophie", elles me semblent tout de même marginalisées dans l'appareil conceptuel. D'autre part, et c'est tout le paradoxe, les philosophies déconstituantes occupent une place de choix dans le travail produit par l'ADPhi, et par Cossutta en particulier, depuis trente ans : un ouvrage et plusieurs articles consacrés au scepticisme (1994, 2003, 2018), deux ans de séminaire consacrés à Éperons couronnés par un ouvrage collectif (Lire Derrida? 2015a), et une préoccupation toujours renouvelée pour les marges du philosophique et du littéraire (2005), comme si la supposée marginalité de certains discours philosophiques était en fait au cœur de l'ADPhi ${ }^{12}$. Ce paradoxe remet ainsi en doute ce qui fait la caractéristique de «la philosophie» pour l'ADPhi, soit qu'« un dispositif énonciatif fonde, de manière en quelque sorte performative, sa propre possibilité, tout en faisant comme s'il tenait cette légitimité d'une source qu'il ne ferait qu'incarner (le Verbe révélé, la Raison, la Loi) » (Maingueneau et Cossutta 1995 : 118). La philosophie apparaît autant composée de discours constituants que de discours destituants, et sans le dire, Cossutta me semble distinguer très nettement deux traditions philosophiques à considérer sur le même plan: celle des discours constituants, et celle des discours destituants.

12 La lecture de cette distinction en termes de traditions philosophiques est esquissée par Maingueneau. Son approche se distingue quelque peu de celle de Cossutta en ce qu'il conserve le concept de "constituance » intact pour y attacher une distinction entre deux « régimes » de discours, l'un marqué, l'autre non:

un régime dominant, non marqué, qui s'appuie sur la discussion argumentée, l'effacement de la subjectivité au profit d'une subjectivité universelle, la mise entre parenthèses du biographique ou de l'affect...[et] un régime marqué: sceptiques, cyniques de l'Antiquité, plus près de nous des penseurs comme Montaigne, Pascal, Kierkegaard, Stirner, Nietzsche, Derrida, le second Wittgenstein... qui nourrissent leur pensée d'un rejet du régime dominant, exploitant dans une démarche non dialectique les éléments refoulés par le premier et investissant des formats textuels singuliers: essais, fragments, journaux, genres littéraires ou religieux... (Maingueneau $2015: 29-30$ )

13 Plusieurs remarques s'imposent: d'abord, les deux «régimes" distingués par Maingueneau sont présentés symétriquement, contrairement aux discours constituants et destituants chez Cossutta. Les deux discours sont pourtant encore dans un rapport asymétrique dans la mesure où le régime marqué apparaît tributaire du régime non 
marqué jugé « dominant ». Ensuite, la tradition seulement esquissée chez Cossutta se fait plus explicite chez Maingueneau, qui, après en avoir énuméré bon nombre de représentants, poursuit ainsi sa réflexion :

On peut lire d'ailleurs à la lumière de cette distinction marqué / non marqué la généalogie imaginaire de la philosophie occidentale. Celle-ci voit dans l'œuvre de Platon son moment inaugural, où se trace la frontière avec des pensées qui ne sont parvenues que par lambeaux et dont on doute que les auteurs soient de "véritables » philosophes, eux dont la parole mêle inextricablement littérature, religion et philosophie. Mais l'œuvre de Platon elle-même tient pour une bonne part son pouvoir de séduction du fait qu'elle est soutenue par un locuteur, Socrate, qui précisément n'a pas écrit et dont la biographie et « l'œuvre » se confondent. On peut toujours se rassurer en disant que Socrate est seulement le porte-parole de Platon qui rend par là hommage à son maître; mais on peut aussi voir là l'inscription, dès les origines, de la faille qui empêche le discours philosophique d'être Un. (2015 : 30-31)

Si la « distinction marqué / non marqué » peut recouvrir « la généalogie imaginaire de la philosophie occidentale", ce n'est pas seulement parce que, comme l'explique Maingueneau, celle-ci inscrit son moment fondateur dans la philosophie platonicienne qui se distingue des philosophies "impures» («littérature, religion et philosophie » mêlées) qui l'ont précédée. Ce n'est pas non plus seulement en raison de l'hétérogénéité constitutive du discours philosophique, cette "faille qui empêche le discours philosophique d'être Un ». C'est, enfin, que le régime marqué, censément dominé et marginal et qui se présente comme tel - on l'a vu avec Derrida -, est absolument central à la compréhension du discours philosophique occidental. Peut-être peut-on ainsi nuancer l'affirmation selon laquelle les philosophes de cette tradition «investissent philosophiquement des modes d'énonciation qui de prime abord relèvent de la littérature » (Maingueneau $2015: 29$ ) ; ne pourrait-on considérer que cette distinction entre modes d'énonciation philosophique et littéraire découlerait d'une pré-conception de la philosophie comme dominée par un régime non-marqué ? Or, si l'on retrace cette tradition en partant des sceptiques et des cyniques, en passant par Montaigne et Burton, jusqu'à Nietzsche et Derrida, on voit que ces philosophes partagent davantage qu'un réinvestissement de modes d'expression et d'énonciation considérés comme habituellement réservés à la littérature : l'usage de la première personne, l'inscription de la biographie et du corps, l'usage de la fiction. Ils partagent d'abord un positionnement sceptique dont découle ce que Cossutta appelle leur " déconstituance " (2015b), le fait que leur légitimité à parler se constitue à travers une délégitimation de la parole philosophique en général ; ils partagent également la figure parodique du philosophe prophète, vox clamantis in deserto (Zarathoustra, bien sûr, mais aussi le "Democritus Junior » de l'Anatomy of Melancholy), qui ne peut connaître qu'un mode d'énonciation distanciée ${ }^{13}$. Ils partagent enfin, "des formats textuels singuliers" (Maingueneau 2015: 30) ou plutôt une grande diversité de formats textuels, parmi lesquels les « essais » (Montaigne), les « journaux » (Derrida), mais aussi l'épistolaire ( $L a$ carte postale), le poème et la prière (Lucrèce)...

Il apparaît alors que les philosophies déconstituantes, qui ont pour particularité de mettre en scène leur supposée marginalité même, loin de s'élaborer aux marges du discours philosophique, en constituent en fait une tradition majeure. La philosophie de Derrida me semble participer de cette tendance et de cette histoire: son illisibilité montrée n'a rien d'inouï, sa mise en question constante de sa propre légitimité, son rapport paradoxal et irrévérencieux à «la philosophie», etc., construisent en fait sa 
légitimité en l'inscrivant dans une tradition qui va des cyniques à Nietzsche ${ }^{14}$. La prise en compte de l'intertexte et plus généralement des reprises génériques, pourrait contribuer à l'étude des philosophies déconstituantes et à réévaluer leur place dans l'ADPhi.

\section{Une relation affinitaire ? Complicités de l'ADPhi et de la philosophie derridienne}

16 La spécificité de la philosophie de Derrida par rapport à l'ADPhi au regard d'autres philosophies appartenant à la même tradition, réside dans le fait qu'elle en est l'exacte contemporaine. Alors que la marginalisation du régime marqué dans l'arsenal de l'ADPhi pourrait laisser croire à une certaine hétérogénéité entre le corpus derridien et l'ADPhi, une mise en regard révèle en fait des affinités profondes: non seulement l'ADPhi et la philosophie derridienne travaillent avec des notions identiques ou extrêmement proches (celles de bio/graphie, de scène d'énonciation...), mais la seconde peut se lire comme une dramatisation des outils de la première. La comparaison des deux corpus ${ }^{15}$ permet ainsi de relativiser l'illisibilité derridienne, et d'offrir à l'ADPhi une perspective légèrement différente de la tradition, foucaldienne, dont elle se revendique plus volontiers.

D'abord, Derrida comme l'ADPhi accordent une place particulière à la biographie du philosophe, ou au biographèm $\mathrm{e}^{16}$. Maingueneau, dans La philosophie comme institution discursive, insiste ainsi sur la nécessité d'intégrer à l'appréhension du discours philosophique une réflexion sur la «biographie des philosophes»: contre la « démarche structuraliste » qui maintiendrait la division entre un « intérieur » du texte et son "extérieur", l'ADPhi cherche à "prendre en compte la biographie des philosophes » dans la constitution du discours philosophique comme tel (2015: 53 et seq.). Derrida quant à lui, non content d'inscrire la biographie des philosophes qu'il commente dans ses textes (en reproduisant des pans entiers de la correspondance de Hegel dans Glas, par exemple), fait de son propre discours un discours autobiographique. Lisant Derrida dans la perspective des catégories de l'ADPhi, celles-ci semblent alors mises en scène, à la fois tenues à distance et dramatisées. Outre ceux qui entremêlent explicitement commentaire philosophique et autobiographie, comme "Circonfession" (Bennington 1991) ou Le Monolinguisme de l'autre (Derrida 1996), certains textes s'attachent à mettre en scène l'inscription du nom propre dans le discours philosophique. Glas est de ceux-là, qui reproduit, par exemple, la signature de l'auteur, face à des lettres de Victor Cousin à Hegel, "pour remarquer le caractère cynique du paraphe » (1974: 208b). La signature n'est donc pas reproduite simplement, sans un tour supplémentaire dans l'inscription du biographique : on pourrait dire qu'il s'agit d'une inscription montrée du biographique dans le discours philosophique, pour bien indiquer que cette inscription se double toujours d'un procédé qui attire l'attention sur elle (un commentaire métadiscursif, par exemple, comme c'est le cas ici).

Ensuite, on peut lire chez Derrida la dramatisation de ce que Maingueneau appelle "scène d'énonciation » $(2015)^{17}$. Tel texte s'analysera en trois scènes distinctes, qui toutes participent également de la scène d'énonciation: la scène englobante, qui « correspond » au «type de discours » utilisé (2015: 66) ; la scène générique, qui définit un horizon d'attente auprès du lecteur d'après le genre du texte (67-70); la scénographie, définie comme "mise en scène singulière de l'énonciation", et qui 
désigne la façon dont « l'énonciateur aménage à travers son énonciation la situation à partir de laquelle il prétend énoncer " $(2015: 70)$. On voit aisément les avantages d'une telle approche: le texte philosophique est alors véritablement considéré comme discours, à la croisée de l'individuel et du social.

Essayant d'appliquer ces outils au corpus derridien, l'analyste se trouve dans une situation inconfortable: ils sont à la fois parfaitement exogènes au corpus (ils ne relèvent pas d'une même conception du texte et de l'écriture, par exemple), et étonnamment adaptés. Ainsi les textes de Derrida se désignent-ils eux-mêmes volontiers comme "scène ». Outre "Freud et la scène de l'écriture" (1967) qui l'annonce dès son titre, Glas se présente comme une scène de théâtre sur laquelle défilent des personnages: Antigone (Derrida 1974: 164a), «le père" (1974: 193b), «Pépé » (1974: 198b) mais aussi Ædipe "cité à comparaître " (1974: 193a), la scène théâtrale se muant alors en tribunal. L'ouverture même du texte met en scène l'espace commun du texte: "quoi du reste aujourd'hui, pour nous, ici, maintenant, d'un Hegel? » dramatise le hic et nunc propre à la scène de lecture par son insistance sur les repères déictiques qui saturent la phrase. Alors que Maingueneau vante l'intérêt de la polysémie du terme de scène "de pouvoir référer à la fois à un cadre et à un processus " (65), Derrida joue carrément des sens multiples de « scène » :

Pourquoi faire passer un couteau entre deux textes? Pourquoi, du moins, écrire deux textes à la fois? Quelle scène joue-t-on? Que désire-t-on? Autrement dit, de quoi a-t-on peur? qui ? de qui? On veut rendre l'écriture imprenable, bien sûr (1974: 76b).

C'est la scène même de l'écriture qui est dramatisée comme scène : scène au carré, scène de scène, le texte derridien semble alors toujours avoir un coup d'avance sur les outils de l'analyse du discours. Il ne s'agit pas d'affirmer que le discours derridien est un discours verrouillé de l'intérieur, inanalysable, qui aurait produit une énonciation complexe en même temps que les outils pour l'analyser. Mais plutôt: le discours derridien est construit pour produire l'effet d'un discours verrouillé, « imprenable », et d'autant plus imprenable que ses conditions d'énonciation y sont mises en scène. La complicité des notions utilisées par l'ADPhi et par Derrida pour qualifier l'énonciation philosophique montre que ces deux régimes discursifs entrent en résonance l'un avec l'autre, et que l'ADPhi est légitime à prétendre analyser le discours derridien comme la philosophie derridienne peut venir enrichir l'arsenal méthodologique de l'ADPhi.

\section{Pour une notion de ton en analyse du discours philosophique}

21 Pour finir, j'aimerais esquisser un passage possible du corpus derridien à l'ADPhi. La notion de ton est quasiment absente des outils de l'ADPhi ${ }^{18}$. Maingueneau y fait référence à deux reprises dans l'introduction au chapitre qu'il consacre à l'ethos. Il insiste d'abord sur la nécessité d'ajouter à la catégorie de scénographie, celle d'ethos, afin d'analyser le « ton » adopté par les énonciateurs. Il poursuit :

Les philosophes privilégient tout naturellement la cohérence de leur argumentation, mais l'autorité qui émane de leur énonciation résulte pour une part d'une correspondance réussie entre le dit et sa source, à travers une scénographie et la manière de dire qu'elle implique. Le déploiement textuel de la doctrine ne fait ainsi qu'un avec l'élaboration de l'instance qui le porte : l'énonciation doit à la fois produire la doctrine et l'instance qui en est le garant. Ce qui fait que le texte a 
besoin de trouver le "ton juste", l'ethos qui lie comme il convient un contenu et une certaine figure de l'énonciateur. (2015:93) philosophique, dans son commentaire de Kant, D'un ton apocalyptique adopté naguère en philosophie (Derrida 1983). Dans ce texte, il montre que ce qui est en jeu derrière la critique kantienne des «mystagogues » qui se prennent pour des grands-seigneurs, c'est bien l'essence de la philosophie :

Alors nos mystagogues jouent du fantôme et du voile, ils remplacent les évidences et les preuves par des "analogies", des "vraisemblances" (Analogien, Wahrscheinlichkeiten); ce sont leurs mots, Kant les cite et nous prend à témoin : vous voyez bien, ce ne sont pas de vrais philosophes, ils recourent à des schèmes poétiques. Tout ça, c'est de la littérature (Derrida $1983: 45$ ).

Et ce sont ces «schèmes poétiques » qui autorisent, croient-ils !, ces faux philosophes, ces « nouveaux prédicateurs » à « se donner de grands airs, [à] occuper par simulacre et mimique la place des grands, [à] usurper ainsi un pouvoir d'essence symbolique " (1983 : 49). C'est parce qu'ils pervertissent la philosophie au point de risquer sa mort que ces ennemis de la vérité se permettent de prendre un «ton grand-seigneur». Le ton marque bien l'endroit de ce que Maingueneau appelle une « faille » $(2015: 31)$ et qui divise irrémédiablement et depuis toujours le discours philosophique en deux : régime non marqué et régime marqué, tradition constituante et tradition déconstituante. Mais il ne le marque pas seulement d'un côté ; comme le souligne Derrida,

Bien que [...] la différence tonale ne passe pas pour essentiellement philosophique, ce n'est pas pour Kant le fait qu'il y ait du ton, de la marque tonale, qui annonce à lui seul la mort de toute philosophie. C'est tel ton, une certaine inflexion socialement codée pour dire telle et telle chose déterminée (1983:21).

Autrement dit : il y a du ton dans le régime marqué comme dans le régime non marqué, chez Kant comme chez Derrida. Comme il y de l'ethos. Alors que l'ethos désigne la façon dont l'énonciateur élabore son image à travers sa manière de dire, le ton pourrait désigner la manière de dire sans qu'elle soit directement raccordée à la constitution d'une image de soi. Cela permettrait, par exemple, de repérer des changements de ton qui ne sont pas forcément les corollaires d'un changement d'ethos. En outre, la notion de ton permettrait de relire la tradition déconstituante à nouveaux frais. Il faut $\mathrm{y}$ insister: le ton recouvre le partage du discours philosophique en une tradition dominante qui valoriserait une énonciation blanche, et une tradition aux marges de la philosophie, dont j'ai essayé de montrer plus haut qu'elle était pourtant tout aussi centrale que la première. C'est à une généralisation du conflit mis en scène par Kant à l'échelle de l'histoire de la philosophie qu'invite Derrida :

Kant parle de la modernité, et des mystagogues de son temps, mais vous aurez vite perçu au passage, sans même que j'aie besoin de désigner explicitement, de nommer ou de tirer tous les fils, à combien de transpositions on pourrait se livrer du côté de notre dite modernité. Je ne dirai pas qu'aujourd'hui chacun se reconnaîtrait de tel ou tel côté, purement et simplement. Mais je suis sûr qu'on pourrait le démontrer, tout discours un peu organisé se trouve ou prétend se 
trouver aujourd'hui des deux côtés, alternativement ou simultanément, même si cet emplacement n'épuise rien, ne fait pas le tour ou le contour de la place et du discours tenu. Et cette inadéquation, toujours elle-même limitée, indique sans doute la plus dense difficulté. Chacun de nous est le mystagogue et l'Aufklärer d'un autre (Derrida $1983: 53$ ).

Plus que de recouvrir l'histoire de la philosophie selon les deux lignes déjà démarquées, la notion de ton permet d'en relativiser l'écart.

\subsection{Le ton de l'écriture}

Plus qu'engager à porter « attention au ton » (Derrida $1983: 18$ ), l'œuvre de Derrida, et tout particulièrement les textes stéréographiques, invitent à retravailler la notion de ton à travers l'élaboration d'une circulation inouïe entre écriture et oralité. Ainsi de "La double séance ", qui, dès ses premières pages, annonce la mise en jeu complexe des notions d'écrit et d'oral:

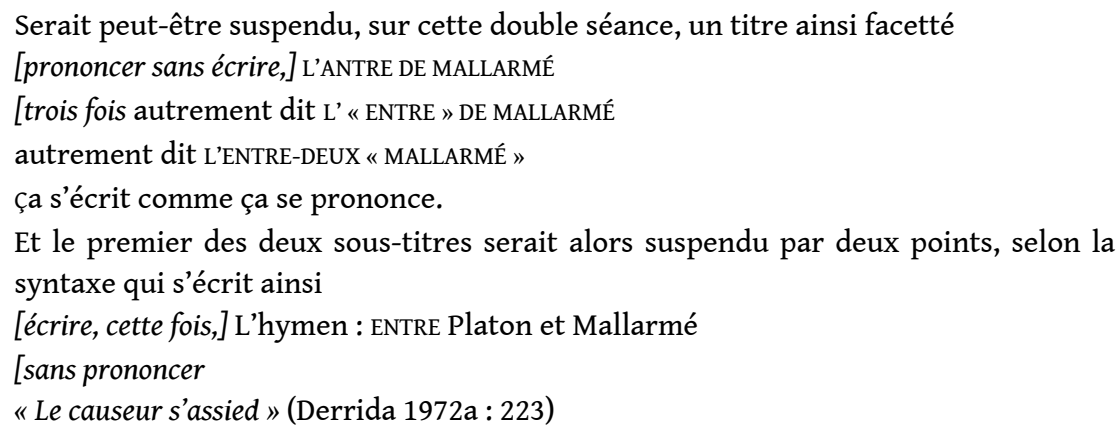

Le jeu sur l'homophonie du titre fonctionne ici doublement ; sous le premier jeu, celui évident qu'on nous donne ici à lire, et qui fut d'abord donné sous forme de conférence au Groupe d'Études théoriques en 1969, on décèle un second jeu, plus complexe : celui que met en scène l'écrivain qui publie ladite conférence. Plutôt que de revoir son texte pour la publication dans le sens d'une traduction des effets oraux (l'homophonie des syntagmes « antre de ", « "entre" de » et " entre-deux »), Derrida choisit d'y exposer la mise en scène de l'écriture et de l'oralité, à l'aide de didascalies véritables, comme en témoigne l'italique. Le jeu homophonique serait bien vain ici, s'il ne fonctionnait également comme un moyen d'inscrire l'indécision de la voix dans l'écrit. Le geste homophonique est en effet immédiatement suivi du jeu inverse : «écrire, cette fois, [...] sans prononcer ». Il s'agit moins alors de réinvestir l'écriture avec des marques d'oralité que d'élaborer une circulation entre écrit et oral, oral et écrit.

«Survivre » s'ouvre sur la même circulation: dans la partie supérieure de la page, se déroule un texte qui multiplie les marques d'oralité, tandis que le quart inférieur est occupé par un "Journal de bord», avec des entrées datées. Le texte dans la partie supérieure commence ainsi :

Mais qui parle de vivre?

Autrement dit sur vivre? (Derrida 1986b : 119)

L'oralité est marquée par l'emploi du verbe "parler » et le début in medias res avec la conjonction « mais »; l'énonciateur semble réagir directement au titre, «Survivre », et, au choix, s'en étonner, ou s'en scandaliser. La même locution adverbiale que dans « La double séance ", " autrement dit », permet d'introduire un déplacement du sens dans la deuxième phrase, qui explicite le lien entre le titre et la question liminale : le «sur » de "survivre » est détaché du verbe et remotivé pour signifier «à propos de ». Comme 
dans l'exemple précédent, c'est l'inscription de l'oralité dans l'écriture qui permet de rendre «l'énoncé [...] indéterminé, mobile, difficile à arrêter » (idem). La bande qui court dans le quart inférieur de la page forme un contre-point à ce début oralisant: le "Journal de bord» s'ouvre en effet sur une dédicace à l'ami Jacques Ehrmann, dont l'énonciateur rappelle qu'il « avait la chance de signer J.E. de ses initiales » (idem). Au jeu homophonique de la partie supérieure de la page répond le jeu homographique des lettres «J.E.»; le texte met ainsi en circulation jeux homophoniques et homographiques, qui se prêtent tantôt à l'œil tantôt à l'oreille, et questionnent l'étanchéité des frontières entre écrit et oral en se rassemblant sur une même page.

31 C'est peut-être dans Feu la cendre qu'on trouve le geste le plus paradoxal concernant ces questions et la mise en circulation la plus étonnante des notions d'écriture et d'oralité. En effet, le texte stéréographique - polylogue sur la page de droite, citations tirées de l'œuvre de Derrida sur la page de gauche - a été mis en voix par Derrida et Carole Bouquet. Dès son «Prologue », Feu la cendre apparaît comme la tentative de travailler une impossible co-inscription de la voix et de l'écriture :

Sur la page, en effet, deux écrits se font face : d'une part, à droite, le polylogue proprement dit, un enchevêtrement de voix en nombre indéterminé, dont certaines paraissent masculines, d'autres féminines, et cela se marque parfois dans la grammaire de la phrase. Ces signes grammaticaux sont lisibles mais ils disparaissent pour la plupart à l'audition, ce qui aggrave une certaine indécision entre l'écriture et la voix, indécision dont le mot là, avec ou sans accent, dans il y a là cendre, faisait déjà courir le risque.

Cette tension risquée entre l'écriture et la parole, cette vibration de la grammaire à la voix, c'est aussi l'un des thèmes du polylogue. Celui-ci, semble-t-il, se destinait à l'œil, il ne s'accordait qu'à la voix intérieure, une voix absolument basse.

Mais par là même il donnait à lire, il analysait peut-être ce qu'une mise en voix pouvait appeler et à la fois menacer de perdre, une profération impossible et des tonalités introuvables. Oserai-je dire de mon désir qu'il avait lieu, son lieu, entre cet appel et cette menace? Qu'attendait-il ?

(Derrida $1987: 8-9$ )

D'un côté, des « Animadversiones » qui se prêtent à la lecture silencieuse, de l'autre un « polylogue » écrit en vue de sa profération, et au cœur de ce dispositif, une phrase, « il y a là cendre ", dont l'indécidabilité repose sur une double inscription grammophonique. Tandis que l'oreille entendra plus volontiers "il y a la cendre ", l'œil lit «il y a là cendre", une possibilité effaçant l'autre. Si l'on pourrait d'abord croire qu'un tel dispositif tend à une réconciliation de la voix et de l'écriture, à une adéquation de la profération au signe, Feu la cendre réinscrit en fait de la différance dans la voix comme dans l'écriture.

Si les «Animadversiones» semblent d'abord impossible à proférer, ce n'est pas seulement parce qu'elles sont des citations de textes; c'est aussi parce qu'elles interviennent en même temps que le polylogue. La mise en voix doit, à cause de sa linéarité même et sous peine de cacophonie, différer cette simultanéité : ainsi des « Animadversiones II » qui n'interviennent qu'à la toute fin de la page de droite dans la version enregistrée (Derrida 1987 : 26-27), avant que cette différance ne s'inverse au profit des «Animadversiones » même, qui se poursuivent sur six pages (et plusieurs minutes), tandis que le polylogue ne présente plus qu'une page blanche. Le procédé de différance, différence et diffèrement, qu'on assigne intuitivement à la mise en voix d'un dispositif stéréographique, apparaît donc également à l'œuvre dans l'écriture de la voix (le polylogue). 


\subsection{Vers une re-définition du ton?} de ton? Je propose de lire dans ces procédés la mise en œuvre du ton tel qu'il est défini dans D'un ton apocalyptique adopté naguère en philosophie: comme une "vibration différentielle pure » (Derrida 1983: 69). Le ton serait précisément cette différance inhérente à l'écriture comme à la parole, qui fait que ni l'une ni l'autre ne sont pures ni exemptes de l'autre. Nulle écriture sans vocalité, nulle voix sans écriture : telle serait le chiasme sur lequel fonder la notion de ton. Cela éviterait au moins un écueil: reconduire une vocalité fantasmée, la voix originaire déconstruite par Derrida, dans l'analyse du discours. Considérer que le ton désigne l'inscription réciproque de la voix et de l'écriture permet de poser, au seuil de l'analyse, une hétérogénéité sauve de tout fantasme de pureté. Il s'agira, pour l'analyste, d'examiner les modulations de cette hétérogénéité, les façons dont ce chiasme initial se configure, en rapport avec l'élaboration du discours philosophique.

Alors que les textes de Derrida pourraient au premier abord sembler échapper aux outils de l'analyse du discours philosophique, ils entretiennent au contraire avec elle des liens affinitaires privilégiés. À travers l'examen de notions centrales dans le champ de l'analyse du discours philosophique (celles de constituance, de constitution et leurs corollaires destituance et destitution; la distinction régime marqué / régime non marqué), j'ai montré comment cette méthode elle-même pouvait parfois reconduire la marginalité montrée des textes les plus atypiques de Derrida. Insister au contraire sur les affinités entre l'analyse du discours philosophique et certains aspects du discours derridien pourrait permettre un échange dynamique entre le corpus derridien et la méthode de l'analyse du discours philosophique. En effet, l'œuvre derridienne n'a de cesse de problématiser sa propre appartenance au champ de la philosophie ; plutôt que de reproduire la marginalisation qu'elle met en scène, je propose de la reconsidérer à travers la notion de ton. Comme je l'ai montré, le ton tel qu'il est développé dans D'un ton apocalyptique recouvre la distinction entre régime marqué et régime non marqué, tout en la déplaçant légèrement. En effet, comme l'écrit Derrida, chacun de nous est «le mystagogue et l'Aufklärer d'un autre» (1983: 53). Deux pistes de travail s'ouvrent alors : d'une part, inscrire le discours philosophique et son « ton » dans une perspective historique, ce qui permettrait justement de déterminer de qui il est le mystagogue et l'Aufklärer à la fois; d'autre part, au niveau du discours lui-même, explorer les modulations de ton au fil de l'élaboration du discours philosophique à travers les diverses configurations du chiasme voix /écriture. Une telle entreprise reviendrait tout à la fois à relativiser les marges de l'ADPhi et à tirer profit des affinités entre l'ADPhi et l'œuvre derridienne. 


\section{BIBLIOGRAPHIE}

Angermuller, Johannes. 2013. Le champ de la théorie : essor et déclin du structuralisme en France, trad. Rosine Inspektor (Paris : Hermann)

Attridge, Derek \& Jacques Derrida. 1992. “"This Strange Institution Called Literature”. An Interview with Jacques Derrida', Attridge, Derek (ed.). Acts of Literature (New York; London: Routledge), 33-75

Bennington, Geoffrey. 1991. Jacques Derrida (Paris : Seuil)

Cavell, Stanley. 1994. A Pitch of Philosophy. Autobiographical Exercises, The Jerusalem-Harvard Lectures (Cambridge, Mass: Harvard U. P.). [Cavell, Stanley. 2003. Un ton pour la philosophie, trad. Sandra Laugier (Paris : Bayard)]

Cossutta, Frédéric. 1994. Le scepticisme, Que sais-je ? (Paris : PUF)

Cossutta, Frédéric. 1995. « Pour une analyse du discours philosophique », Langages 119, «L'analyse du discours philosophique », 12-39

Cossutta, Frédéric. 2003. « Pour une critique sceptique de la pragmatique transcendantale de K. O. Appel », Methodos. Savoirs et textes 3, « Figures de l'irrationel », [En ligne] DOI : https://doi.org/ $10.4000 /$ methodos.118

Cossutta, Frédéric. 2005. « Discours philosophique, discours littéraire : le même et l'autre », Rue Descartes 50, « L'écriture des philosophes », 6-20

Cossutta, Frédéric. 2015a. « Éperons. Les styles de Nietzsche. Un texte indéchiffrable?», Maingueneau, Dominique \& Mathilde Vallespir (éds.). Lire Derrida?, Le Discours philosophique, (Limoges : Éditions Lambert-Lucas), 13-41

Cossutta, Frédéric. 2015b. « Les Discours constituants vingt ans après », Angermüller, Johannes \& Gilles Philippe, analyse du discours et dispositifs d'énonciation. Autour des travaux de Dominique Maingueneau (Limoges : Lambert-Lucas), 61-70

Cossutta, Frédéric. 2018. « Mystique et scepticisme », Le Philosophoire 49, 43-80

Cossutta, Frédéric, Delormas, Pascale \& Dominique Maingueneau (éds.). 2012. La vie à l'œuvre. Le biographique dans le discours philosophique (Limoges : Lambert-Lucas)

Currie, Mark. 2013. The Invention of Deconstruction (Houndmills, Basingstroke, Hampshire: Palgrave Macmillan)

Deleuze, Gilles. 2007. Présentation de Sacher-Masoch : le froid et le cruel (Paris : Minuit)

Derrida, Jacques. 1967. L'écriture et la différence (Paris : Seuil)

Derrida, Jacques. 1972a. La dissémination (Paris : Seuil)

Derrida, Jacques. 1972b. Marges de la philosophie (Paris : Minuit)

Derrida, Jacques 1974. Glas (Paris : Galilée)

Derrida, Jacques. 1983. D’un ton apocalyptique adopté naguère en philosophie (Paris : Galilée)

Derrida, Jacques. 1984. Signéponge /Signsponge, trad. Richard Rand (New York: Columbia U. P.)

Derrida, Jacques. 1986a. Schibboleth Pour Paul Celan (Paris : Galilée)

Derrida, Jacques. 1986b. Parages (Paris : Galilée) 
Derrida, Jacques. 1987. Feu la cendre (Paris : Des Femmes)

Derrida, Jacques. 1988. «Y a-t-il une langue philosophique ? ", Revue Autrement 102, « À quoi pensent les philosophes », 30-37

Derrida, Jacques. 1996. Le monolinguisme de l'autre (Paris : Galilée)

Foucault, Michel. 2005. Les mots et les choses (Paris : Gallimard)

Maingueneau, Dominique. 2015. La philosophie comme institution discursive (Limoges : Éditions Lambert-Lucas)

Maingueneau, Dominique \& Frédéric Cossutta. 1995. "L'analyse des discours constituants ", Langages 117, « Les analyses du discours en France », 112-125

Maingueneau, Dominique \& Mathilde Vallespir (éds.). 2015. Lire Derrida ? (Limoges : Éditions Lambert-Lucas)

Mercier, Thomas Clément. 2018. "Before the Spectres: Reading Derrida's Archive on Materiality \& Ideology”, Derrida Today Conference, 23-26 Mai 2018, Montréal

Musgrave, David. 2014. Grotesque Anatomies: Menippean Satire since the Renaissance (Cambridge: Cambridge Scholars Publishing)

Searle, John R. 1983. “The Word Turned Upside Down”, The New York Review of Books [En ligne], consulté le 11 février 2018. URL: http://www.nybooks.com/articles/1983/10/27/the-wordturned-upside-down/

Smith, Barry et al. 1992. 'Derrida Degree a Question of Honor', The Times (London)

Wiser, Antonin. 2017. «L'espace du style : sur les trois versions d'Éperons $(1973,1976,1978)$ de Jacques Derrida », Genesis 44, [En ligne] mis en ligne le 17 mai 2018, consulté le 06 juillet 2018. URL : http://journals.openedition.org/genesis/1768 ; DOI : 10.4000/genesis.1768

\section{NOTES}

1. J'utilise le terme «stéréographique " pour désigner les textes doubles de Derrida : autant ceux mettant en regard deux colonnes («Tympan», Glas, et dans une moindre mesure «La double séance »), que ceux divisés en deux bandes horizontales ("Survivre », "Circonfession »), que Feu la cendre dont le statut est un peu particulier puisqu'il s'agit de confronter un texte inédit sur la page de droite à une collection de citations extraites des œuvres de Derrida lui-même sur la page de gauche. Le terme apparaît dans « Tympan » : « Où est passé le corps du texte quand la marge n'est plus une virginité secondaire mais une inépuisable réserve, l'activité stéréographique d'une tout autre oreille?» (Derrida 1972 : XIX).

2. Un mot dont il y a une seule occurrence dans un texte/corpus

3. Frédéric Cossutta a plusieurs fois souligné le risque que comportait le discours derridien de provoquer sa propre reproduction; et en effet, il existe bien un « discours épigonal » (Cossutta) derridien, qui imite volontiers les tropes utilisés par le maître ; voir Cossutta 2015a, et un travail en cours intitulé «La voix de son maître» sur les écritures de disciple, https:// gradphi.hypotheses.org/seminaire-2017-2018

4. On peut penser à Mimesis des articulations dont le texte introductif met en scène un «je » dont on comprend qu'il est tour à tour l'un des six signataires du livre (Agacinski, Derrida, Kofman, Lacoue-Labarthe, Nancy, Pautrat), sans qu'on puisse jamais savoir lequel ; le texte exhibe ainsi la mise en œuvre d'un « je » collectif et instable, non référentiel et pourtant auctorial.

5. Sur la génétique d'Éperons, voir Wiser 2017. 
6. Désormais notée ADPhi.

7. On pourrait ajouter Lautréamont dans « Hors Livre » (Derrida 1972a), Philippe Sollers dans « La Dissémination » (1972a), Valéry dans « Qual Quelle » (1972b), Celan dans Schibboleth (1986a).

8. La philosophie de Derrida a donné lieu à une réception complexe traversée par cette division entre philosophie et littérature ; pour une analyse des transformations induites par la réception nord-américaine (et littéraire) de sa philosophie, voir Currie 2013. Certains ont assigné Derrida au champ de la littérature (et plus souvent, de la poésie) de manière polémique et afin de disputer l'appartenance de son œuvre au champ de la philosophie (voir Searle 1983, et Smith et al. 1992).

9. Il ne s'agit pas d'affirmer que l'œuvre de Derrida appartient davantage à la littérature qu'à la philosophie - Derrida s'est souvent expliqué là-dessus, ainsi en 1988, et dans Attridge et Derrida (1992), mais plutôt qu'elle s'exhibe comme posant la question des limites du champ philosophique et ainsi celle de sa propre appartenance à celui-ci.

10. Je me réfère à Littré.

11. C'est par cette articulation du texte et du contexte que l'analyse du discours française et l'analyse du discours philosophique se distinguent des approches structuralistes qui les ont précédées. Dans ce texte comme dans d'autres, les auteurs font du structuralisme un repoussoir. Ici: «On ne cherchera donc pas, comme dans la démarche structuraliste, une théorie de "l'articulation" entre le texte et une réalité muette, non-textuelle: cela reviendrait à présupposer le partage même qu'on cherche à surmonter» (Maingueneau \& Cossutta 1995 : 114-115).

12. On pourrait dire que Cossutta marginalise dans son travail d'analyse du discours ce qu'il met au centre de son travail de philosophe.

13. Pour une étude de la parole prophétique en philosophie dans les années 1960, voir Angermuller 2013.

14. Plusieurs ont déjà soutenu l'hypothèse selon laquelle Derrida reprenait, notamment dans ses textes stéréographiques, le genre - cynique - de la satire ménippée (voir en particulier Musgrave 2014) ; dans un travail en cours, je tente de démontrer avec précision dans quelle mesure Derrida s'inscrit dans ce genre hérité du cynisme.

15. Précisons qu'il ne s'agit en aucun cas de réduire la spécificité de chacun des corpus, ni de nier leurs différences disciplinaires et herméneutiques évidentes.

16. Le GradPhi a ainsi consacré plusieurs années de son séminaire à cette question, travaux résumés dans un ouvrage collectif intitulé La vie à l'œuvre. Le biographique dans le discours philosophique (Cossutta, Delormas \& Maingueneau 2012).

17. La notion "permet d'éviter des notions telles que celles de "situation d'énonciation" (les coordonnées des repérages déictiques), qui est d'ordre strictement linguistique, ou celle de “ situation de communication", qui peut être utilisée dans une approche purement sociologique où l'activité de parole est décrite en quelque sorte de l'extérieur » (Maingueneau 2015 : 65).

18. C'est une notion peu discutée, à l'exception, dans un tout autre contexte, de Cavell 1994.

\section{RÉSUMÉS}

Alors que l'Analyse du discours vise à forger des catégories permettant de lire et de décrire les opérations discursives dans les œuvres de philosophie, certains textes semblent résister à 
l'analyse. C'est le cas de certains livres de Derrida comme Glas (1974) ou Feu la cendre (1986), textes en deux colonnes dont l'auteur lui-même a pu dire qu'ils n'étaient «ni [...] philosophique[s], ni [...] poétique[s]» (Lévesque et McDonald $1982: 186$ ). Plutôt que de céder à l'apparente illisibilité de ces textes, et ainsi de conclure à l'impuissance de l'Analyse du discours philosophique à les analyser, cet article propose de relativiser la supposée incompatibilité de l'analyse du discours philosophique et du corpus derridien. Après avoir souligné les affinités entre les deux corpus, ce travail tentera de redéfinir la notion de ton à partir de D'un ton apocalyptique adopté naguère en philosophie et de Feu la cendre, en vue de son emploi dans le champ de l'analyse du discours philosophique.

If discourse analysis sets itself to devise useful categories to read and describe discursive operations at work in philosophical discourses, some texts seem to resist analysis. Glas (1974 [1986]) and Feu la cendre [Cinders] (1986 [2004]), two-columned texts of which the author himself said that they were "neither [...] philosophical, nor [...] poetical" (Lévesque \& McDonald 1982: 186), seem to fall in that category. Rather than giving in to these texts' apparent illegibility, and thus concluding that philosophical discourse analysis simply can't analyse them, this article offers to put the supposed incompatibility between philosophical discourse analysis and the Derridean corpus in perspective. Once emphasised the commonalities between the two corpora, we will redefine the notion of "tone" drawing on D'un ton apocalyptique adopté naguère en philosophie ["Of An Apocalyptic Tone Recently Adopted in Philosophy"] (Derrida 1983 [1984]) and Feu la cendre, in order to make this notion useful within the field of philosophical discourse analysis.

\section{INDEX}

Keywords : constituent discourse, Derrida, methodology, philosophical discourse analysis, scenography

Mots-clés : analyse du Discours philosophique, Derrida, discours constituant, méthodologie, scénographie

\section{AUTEUR}

\section{CHARLOTTE THEVENET}

University College London 